\title{
PEDOT:PSS Interfaces Support the Development of Neuronal Synaptic Networks with Reduced Neuroglia Response In vitro
}

\author{
Giada Cellot ${ }^{1 * \dagger}$, Paola Lagonegro ${ }^{2 \dagger}$, Giuseppe Tarabella ${ }^{2}$, Denis Scaini ${ }^{3,4}$, Filippo Fabbri ${ }^{2}$, \\ Salvatore lannotta ${ }^{2}$, Maurizio Prato ${ }^{5}$, Giancarlo Salviati ${ }^{2}$ and Laura Ballerini ${ }^{1,4 *}$ \\ ${ }^{1}$ Department of Neuroscience, International School for Advanced Studies, Trieste, Italy, ${ }^{2}$ IMEM-CNR Istituto, Parma, Italy, \\ ${ }^{3}$ ELETTRA Synchrotron Light Source, Trieste, Italy, ${ }^{4}$ Department of Life Science, University of Trieste, Trieste, Italy, \\ ${ }^{5}$ Department of Chemical and Pharmaceutical Sciences, University of Trieste, Trieste, Italy
}

OPEN ACCESS

Edited by:

Michele Giugliano,

University of Antwerp, Belgium

Reviewed by:

Arti Ahluwalia

University of Pisa, Italy

Orit Shefi,

Bar llan University, Israel

${ }^{*}$ Correspondence:

Giada Cellot

cellot@sissa.it;

Laura Ballerini

laura.ballerini@sissa.it orcid.org/0000-0001-8420-0787

${ }^{\dagger}$ These authors have contributed equally to this work

Specialty section This article was submitted to Neural Technology,

a section of the journal

Frontiers in Neuroscience

Received: 01 October 2015 Accepted: 24 December 2015 Published: 14 January 2016

Citation:

Cellot G, Lagonegro P, Tarabella G,

Scaini $D$, Fabbri F, lannotta $S$,

Prato M, Salviati $G$ and Ballerini $L$ (2016) PEDOT:PSS Interfaces Support the Development of Neuronal Synaptic

Networks with Reduced Neuroglia

Response In vitro.

Front. Neurosci. 9:521. doi: 10.3389/fnins.2015.00521
The design of electrodes based on conductive polymers in brain-machine interface technology offers the opportunity to exploit variably manufactured materials to reduce gliosis, indeed the most common brain response to chronically implanted neural electrodes. In fact, the use of conductive polymers, finely tailored in their physical-chemical properties, might result in electrodes with improved adaptability to the brain tissue and increased charge-transfer efficiency. Here we interfaced poly(3,4-ethylenedioxythiophene):poly(styrene sulfonate) (PEDOT:PSS) doped with different amounts of ethylene glycol (EG) with rat hippocampal primary cultures grown for 3 weeks on these synthetic substrates. We used immunofluorescence and scanning electron microscopy (SEM) combined to single cell electrophysiology to assess the biocompatibility of PEDOT:PSS in terms of neuronal growth and synapse formation. We investigated neuronal morphology, density and electrical activity. We reported the novel observation that opposite to neurons, glial cell density was progressively reduced, hinting at the ability of this material to down regulate glial reaction. Thus, PEDOT:PSS is an attractive candidate for the design of new implantable electrodes, controlling the extent of glial reactivity without affecting neuronal viability and function.

Keywords: PEDOT:PSS, hippocampal neurons, glial cells, neural interfaces, conductive polymers, single cell patch clamp

\section{INTRODUCTION}

Brain-machines interfaces are prosthetic devices designed to ameliorate the prognosis of neurological patients by targeting non-specific sensory/motor deficits in brain injuries, or more specific symptoms in Parkinson's disease, chronic pain and epilepsy (Donoghue, 2002; Machado et al., 2010; Goodrick, 2014).

Recording/stimulation electrodes are the core component of implanted interfaces and need to show excellent and stable electrical properties together with a good biocompatibility once chronically exposed to the biological environment.

The most common cause of implant failure is the brain tissue reaction against the electrodes (Polikov et al., 2005). This reaction favors the glial-scar formation leading to devices with high impedance and poor electrical charge transfer (Lempka et al., 2009). 
The physical and chemical properties of conducting polymers (CPs) allow the development of devices characterized by improved adaptation to the brain tissue (Green et al., 2008; Williamson et al., 2015). Together with adaptability, CP electrodes are characterized by low impedance when interfaced to excitable tissues. Both these features improve the quality of the recorded signals (Ludwig et al., 2006) and are required in the design of high-performing electrodes.

CPs is composed by repeating units of covalently bonded monomers organized in highly conductive films when polymerized in the presence of anionic dopants (Heeger, 2001). By varying the production process and the type of dopant it is possible to tune CPs mechanical and electrical performance (Dimitrieva et al., 2009). Within CPs, poly(3,4ethylenedioxythiophene):poly(styrene sulfonate) (PEDOT:PSS) is a suitable candidate for the development of high-performance electrode-coatings toward biomedical applications (Schmidt et al., 1997; Cui et al., 2003; Ludwig et al., 2006; Xiao et al., 2006; Berggren and Richter-Dahlfors, 2007; Richardson-Burns et al., 2007; Collazos-Castro et al., 2010; Forcelli et al., 2012). This is motivated by PEDOT:PSS chemical stability in aqueous environment (Yamato et al., 1995) combined to the ease at which PEDOT:PSS physical features can be tuned. For instance, when PEDOT:PSS is doped with substances of decreasing molecular size, the surface roughness of CP films increases (Baek et al., 2014). A similar effect results from increasing the concentrations of the PSS in the electro polymerization solution (Tamburri et al., 2009).

Primary embryonic neurons were shown to survive once embedded in PEDOT:PSS, (Richardson-Burns et al., 2007) and the long-term adhesion of neuronal cells on PEDOT:PSS substrates was improved by pre-layering the CP surface with poly-lysine (Collazos-Castro et al., 2010).

PEDOT:PSS has been polymerized to coat multi-electrode arrays (MEAs). In these experiments the stimulation delivered by PEDOT:PSS layered electrodes evoked stronger neuronal responses when compared to uncoated ones (Nyberg et al., 2007).

It has been recently shown that adaptable electrode arrays, realized on a thin film of parylene- $\mathrm{C}$ and containing microelectrodes made by PEDOT:PSS can be used for in vivo electrocorticography $(\mathrm{ECoG})$ in rats. In these experiments sharp-wave events mimicking epileptic spikes were successfully recorded (Khodagholy et al., 2011). In a further development, electrochemical transistor (OECT) based on PEDOT:PSS, used to record brain activity in vivo, displayed a superior signalto-noise ratio (SNR) when compared with surface electrodes (Khodagholy et al., 2013).

In this technology area, one of the more advanced results is represented by the NeuroGrid: an organic material-based, ultra-conformable, biocompatible, and scalable neural interface array with neuron-sized-density electrodes, exploited for in vivo recording of superficial cortical activity (Khodagholy et al., 2015).

Notwithstanding these studies, the impact of PEDOT:PSS interfaces on neuronal synaptic activity and glial-cell reactivity has not been fully investigated. This is a relevant issue, in particular when CPs electrodes are involved in the design of long-term implants (Lempka et al., 2009; Gunasekera et al., 2015).
In the present study we investigated the biocompatibility of PEDOT:PSS layers when challenged with postnatal brain cells (neurons and neuroglia) to optimize their use in future prosthetic devices. The electrical and morphological properties of PEDOT:PSS doped with different concentrations of ethylene glycol (EG) were analyzed. To assess the viability and performance of neurons and synaptic networks we developed short- and long-term hippocampal cultures (i.e., 1 or 3 weeks in vitro) and we performed single cell electrophysiology, scanning electron microscopy (SEM) and immunofluorescence to document synaptic activity, cell morphology and density when interfaced to PEDOT:PSS films. We investigated glial reactivity to PEDOT:PSS substrates by immunofluorescence technique.

\section{MATERIALS AND METHODS}

\section{Preparation of PEDOT:PSS Layers}

Poly(3,4-ethylenedioxythiophene):poly(styrenesulfonate), termed PEDOT:PSS, type Clevios PH1000 was purchased from Heraeus Conductive Polymers Division.

Successively PEDOT:PSS solution was firstly doped with a 0.05 vol. of dodecyl benzene sulfonic acid (DBSA) surfactant (Sigma Aldrich) for efficient film forming, and then different concentrations $\mathrm{v} / \mathrm{v}(0,1$, and $3 \%)$ of EG were added to enhance electrical conductivity with respect to the pristine state (Crispin et al., 2003; Ouyang et al., 2004; Romeo et al., 2015). One solution was prepared without EG addition and used as reference control.

Homogeneous PEDOT:PSS films at different EG concentrations were spin coated on a glass slides of $1 \times 3 \mathrm{~cm}$ at $1500 \mathrm{rpm}$ for $30 \mathrm{~s}$. The final films thickness was around $80 \mathrm{~nm}$, as measured with the profilometer (Tarabella et al., 2013). Slides were finally baked on a hot plate at $140 \mathrm{C}$ for $60 \mathrm{~min}$.

\section{Electrical Characterization of PEDOT:PSS Layers}

Electrical measurements of PEDOT:PSS layer were obtained by 2-point source/measure precision unit (Agilent B2902A) and controlled by homemade LabView software. The I-V characteristics are acquired, after the realization of Silver Ohmic contacts distant $1 \mathrm{~cm}$, spanning the applied voltage from $-1 \mathrm{~V}$ to $+1 \mathrm{~V}$ achieved in $0.1 \mathrm{~V}$ steps and recording the resulting current flowing in the PEDOT:PSS layer. Different measurements were realized with different EG doping levels of PEDOT:PSS.

\section{Contact Angle}

Five micro liter of water were dropped on the surfaces and the images captured using a homemade static contact angle measuring system. The equilibrium contact angle was evaluated by a statistical study of several images (Chen et al., 2005): two layers where investigated for each EG concentration and the measurement were repeated three times for each sample.

\section{Atomic Force Microscopy (AFM)}

AFM was used for studying at high-resolution the threedimensional reconstructions of PEDOT:PSS layers at three different EG doping conditions (0, 1, and 3\%). All AFM images 
were acquired using a commercially available microscope (Solver Pro AFM from NT-MDT-NT-MDT Co.-Moscow-Russia) endowed with a closed-loop scanner. Measurements were carried out in air at room temperature working in dynamic mode. Cantilevers, characterized by a resonant frequency of $90 \mathrm{kHz}$ and a force constant of $1.74 \mathrm{nN} / \mathrm{nm}$ (NSG03 series from NTMDT_NT-MDT Co.-Moscow-Russia) were used working at low oscillation amplitudes with half free-amplitude set point. High-resolution images were $512 \times 512$ pixels frames acquired at 1 lines/s scan speed working in air. All AFM data were analyzed using Gwyddion (Nečas and Klapetek, 2013), scanning probe microscopy data analysis free software. Surface roughness was computed as the root-mean-square (RMS) value of the height irregularities of AFM images. The ratio between the surface area and the projected surface area, $\mathrm{S}_{r}$, was computed from AFM images by simple triangulation and correlated to roughness values.

\section{Ethical Approval}

All experiments were performed in accordance with the European Community Council Directive of 24 November 1986 (86/609EEC) and Italian law (decree 26/14) and were approved by local Authority Veterinary Service.

The University of Trieste Animal Facility (Life Sciences Department, Italy, authorized by the Italian Ministry of Health) hosted animals and breeding conditions and procedures complied with the 2010/63/UE EU guidelines and Italian law $(26 / 14)$.

Neonatal rats were sacrificed by rapid decapitation and the tissue of interest (hippocampus) harvested, all efforts were made to minimize suffering. The work was performed on explanted tissue and did not require ethical approval.

\section{Cultures and Immunofluorescence}

Hippocampal neurons were isolated from postnatal (P2-3) rat pups and seeded with a standard amount of cells $(\sim 150,000$ cells/coverslips) as previously reported (Lovat et al., 2005) on different substrates. Cells were plated on glass (control cultures) or PEDOT:PSS (with different concentrations of EG, pretreated with plasma cleaning) coverslips, both previously layered with poly-ornithine (Sigma-Aldrich), to increase permissiveness of surfaces. Each coverslip was incubated with $300 \mu \mathrm{l}$ of polyornithine containing solution for a least $1 \mathrm{~h}$, then the drop was removed and cells seeded. Cultured cells were used for experiments at 8-22 days in vitro (DIV).

To quantify neuronal and glial cells density, cultures (control and PEDOT:PSS) were immune-labeled following the procedure previously described (Cellot et al., 2011). Briefly, cultures were fixed with $4 \%$ paraformaldehyde in PBS (20 min), then, upon washout in PBS, incubated in blocking solution and subsequently incubated with rabbit polyclonal antibody against $\beta$ tubulin III (1:250 dilution; Sigma-Aldrich) and mouse monoclonal antibody against GFAP (1:200 dilution; Sigma-Aldrich). Upon washout in PBS, cultures were incubated with the secondary goat anti-rabbit Alexa Fluor 594 (1:500, Invitrogen) and goat anti-mouse Alexa Fluor 488 (1:500, Invitrogen). Two culture series (4 fields for each slide) were sampled for each condition. Samples were visualized with a Leica DM 6000 microscope at 20x magnification. Offline analysis of the images was performed with the open source image-processing package Fiji.

The content of GFAP was estimated by selecting squared areas $\left(25 \mu \mathrm{m}^{2}\right)$ close to the nucleus in randomly chosen glial cells. The mean intensity of fluorescence within this region was calculated using dedicated tool of Fiji software. Background fluorescence, measured in region of sample without cells, was subtracted from fluorescence values (Salazar et al., 2008). Area of single glial cells, whose perimeter was manually drawn, was automatically measured by the software in randomly selected cells, sufficiently isolated from neighbors.

\section{Scanning Electron Microscopy (SEM)}

SEM was used to investigate the morphology of cellular cultures developed on PEDOT:PSS substrates and on controls. Images were acquired collecting secondary electrons on a Gemini SUPRA 40SEM (Carl Zeiss NTS GmbH, Oberkochen, Germany) working at an acceleration voltage of $5 \mathrm{keV}$. Before SEM characterization cellular samples were washed with $0.1 \mathrm{M}$ cacodylate buffer $(\mathrm{pH}=7.2)$ and fixed with a solution containing $2 \%$ glutaraldehyde (Fluka, Italy) in $0.1 \mathrm{M}$ cacodylate buffer for $1 \mathrm{~h}$ at RT. Cultures were then washed in a cacodylate buffer and dehydrated by dipping in water/ethanol solutions at progressively higher alcohol concentrations (50, 75, 90, 95, 98, and 100\% ethanol for $10 \mathrm{~min}$ each). Afterwards samples were let in $100 \%$ ethanol to dry at $4{ }^{\circ} \mathrm{C}$ overnight. Prior to SEM imaging samples were gold metalized in a metal sputter coater (Polaron SC7620). We explore the neuronal morphology in terms of number of neuritis exiting the cell soma. In doing this we sampled in all groups individual neurons clearly isolated from other cells. When investigating the mean number of neuritis departing from the cell soma (Lovat et al., 2005) in these samples we found no significant differences between control and PEDOT:PSS substrates (control: $3 \pm 0.2$, PEDOT 0\%EG: $3.4 \pm 0.3$, PEDOT 1\%EG: $2.9 \pm 0.4$ neurites/cell, $n=8$ for each condition).

\section{Electrophysiology}

A patch-clamp amplifier (multiclamp 700b, Axon Instruments, Sunnyvale, CA, USA) was used to record visually identified (with an upright microscope equipped with differential interference contrast optics and infrared video camera) neurons, using the patch-clamp technique in voltage and current modes. Whole-cell recordings were obtained with pipettes (4-7 M $\Omega$, Hingelberg, Malsfeld, Germany) containing $120 \mathrm{mM} \mathrm{K}$ gluconate, $20 \mathrm{mM}$ $\mathrm{KCl}, 10 \mathrm{mM}$ HEPES, $10 \mathrm{mM}$ EGTA, $2 \mathrm{mM} \mathrm{MgCl}_{2}$, and $2 \mathrm{mM}$ $\mathrm{Na}_{2}$ ATP (pH 7.35 adding $\mathrm{KOH}$ ). The external solution contained the following: $150 \mathrm{~mm} \mathrm{NaCl}, 4 \mathrm{~mm} \mathrm{KCl}, 1 \mathrm{~mm} \mathrm{MgCl}_{2}, 2 \mathrm{~mm}$ $\mathrm{CaCl}_{2}, 10 \mathrm{~mm}$ HEPES, $10 \mathrm{~mm}$ glucose, $\mathrm{pH}$ 7.4. All experiments were performed at $18-22^{\circ} \mathrm{C}$.

Liquid junction potential was $13 \mathrm{mV}$; membrane potential values were not corrected for it. All recordings were performed at $-58 \mathrm{mV}$ of holding potential. Cells exhibiting $>15 \%$ changes in either series resistance or holding were excluded from the analysis. The series resistance was $<20 \mathrm{M} \Omega$ and it was not compensated. 


\section{Data Analysis}

Data were transferred to a computer hard disk after digitization with an A/D converter (Digidata 1322, Molecular Devices). Data acquisition (digitized at $10 \mathrm{kHz}$ and filtered at $2 \mathrm{kHz}$ ) was performed with pClamp 9.2 software (Molecular Devices, Sunnyvale, CA, USA).

In voltage clamp mode, cells were stimulated with a $100 \mathrm{~ms}$ lasting hyperpolarizing stimulus $(10 \mathrm{mV})$, then, in the recording, area below the capacitative transients was measured and normalized for voltage transient amplitude to calculate cellular capacitance; input resistance was obtained through Ohm's law, by measuring the amplitude of steady state current generated by voltage transient. Spontaneous postsynaptic currents were analyzed using pClamp 9 (Molecular Devices, Sunnyvale, CA, USA). This program uses a detection algorithm based on a sliding template. The template did not induce any bias in the sampling of events because it was moved along the data trace by one point at a time and was optimally scaled to fit the data at each position. All the collected events were averaged and the amplitude of current was calculated as that of the mean trace.

Statistical significance was tested using unpaired Student $t$-test (Origin, Northampton, MA, USA). A $p<0.05$ was considered as statistically significant. Values are given as mean \pm SEM.

\section{RESULTS}

\section{Physical and Morphological Analysis of PEDOT:PSS}

PEDOT:PSS layers doped with different amounts of EG were characterized for their physical and morphological properties before using them as growth substrates for culturing hippocampal cells.

The I-V characteristics of PEDOT:PSS layer doped with different concentration of EG, varying from undoped (0\%) up to the $3 \%$ are shown in Figure 1A. The electrical conductance shows an increase of about one order of magnitude, increasing the concentration of EG. In fact, undoped PEDOT:PSS layer shows an electrical conductance of $2.35 \times 10^{-5} \mathrm{~S}$, meanwhile the electrical conductance increases up to $1.5 \times 10^{-4} \mathrm{~S}$ in the case of the $3 \%$ doped films.

The digital images of water equilibrium contact angle of PEDOT:PSS layers doped with different concentration of EG are reported in Figure 1B. The equilibrium contact angle decreases from $21.96^{\circ}$ to $12.10^{\circ}$ with increasing EG from 0 to $3 \%$. The static contact angle analysis reveals that the wettability of the PEDOT:PSS layer increases with the same concentrations of EG used to improve conductance.

AFM measures on $10 \times 10 \mu \mathrm{m}^{2}$ sampled areas show a relatively uniform surface for all samples while, at the nanoscale, surfaces display different granularity (Figure 1C). Roughness analysis reveals values of $1.73,2.38$, and $6.69 \mathrm{~nm}$ for PEDOT:PSS doped with 0,1 , and $3 \%$ EG, respectively. Obtained roughness values are slightly higher than values characterizing cleaned glass substrates (Henke et al., 2002) and similar polymeric materials used for cell development (e.g., polydimethylsiloxane, PDMS; Palchesko et al.,
2012). For a comprehensive description of PEDOT:PSS surface characteristics refer to Kim et al. (2011).

Surface analysis highlighted values of the ratio $\left(S_{r}\right)$ between the effective surface area and the projected surface area of $1.0059,1.0018$, and 1.030 for the three substrates. These values correspond to an increase of the exposed surface of about $0.6,0.2$, and $3 \%$, respectively for 0,1 , and $3 \%$ EG contents, compared to a perfectly flat surface characterized by a unitary value of $S_{r}$.

\section{PEDOT:PSS Layers Supporting the Growth of Hippocampal Cultures}

Upon characterization the EG doped PEDOT:PSS layers were used as growth substrates for hippocampal cultures. Neuronal and glial cells were analyzed at different times of their in vitro development.

\section{Undoped PEDOT:PSS and PEDOT:PSS 1\% EG are Permissive Substrates for Neuronal Cultures}

In a first set of experiments, we investigated whether the EG dopant addition in PEDOT:PSS layers affected short-term hippocampal cultures viability.

Neonatal rat hippocampal cells were seeded on undoped PEDOT:PSS (0\% EG), PEDOT:PSS 1\% EG and control substrates. Cells attached to all the substrates and began extending neurites within the first $24 \mathrm{~h}$. The first set of experiments was performed after $8 \mathrm{DIV}$, a time known to allow for synaptic network development in vitro (Lovat et al., 2005; Mazzatenta et al., 2007; Cellot et al., 2011). Cultures grown on PEDOT:PSS and on control substrates were fixed for immunofluorescence to report cell culture composition and relative cell density. $\beta$-tubulin IIIpositive neurons and GFAP-positive glial cells (Fabbro et al., 2012) are shown in Figure 2A. Neuronal cell density is similar between control and PEDOT:PSS layers ( $388 \pm 40,434 \pm 29$, and $366 \pm 20$ cells $/ \mathrm{mm}^{2}$, respectively control, undoped PEDOT:PSS and PEDOT:PSS 1\% EG, $n=8$ fields each). On the contrary, glial cell density appears reduced on PEDOT:PSS layers with respect to the control $\left(217 \pm 10\right.$ cells $/ \mathrm{mm}^{2}$ in control, $140 \pm 8$ cells $/ \mathrm{mm}^{2}$ on undoped PEDOT:PSS and $185 \pm 15$ cells $/ \mathrm{mm}^{2}$ on PEDOT:PSS $1 \% \mathrm{EG})$, although the difference was statistically significant only between undoped PEDOT:PSS and control $(p<0.005, T$-test; Figure 2B).

We further quantify the intensity of GFAP fluorescence and the dimension (area size) of single glial cells in the different culture groups. No differences were observed in the measured parameters (GFAP fluorescence: control $42 \pm 1.4$ arbitrary units, undoped PEDOT $46 \pm 1.7$ arbitrary units and PEDOT $1 \%$ EG $40 \pm 1.7$ arbitrary units; glial cells area: control $2483 \pm 202 \mu \mathrm{m}^{2}$, undoped PEDOT $2345 \pm 168 \mu \mathrm{m}^{2}$ and PEDOT $1 \%$ EG $2143 \pm 165 \mu \mathrm{m}^{2}, n=43, n=40, n=44$ respectively; Figure 2B).

The viability of neurons grown on PEDOT:PSS layers was assessed by single cell patch clamp recordings. Neuronal passive properties, such as the resting membrane potential, membrane capacitance and input resistance, were measured. These parameters are commonly considered as indicators of 

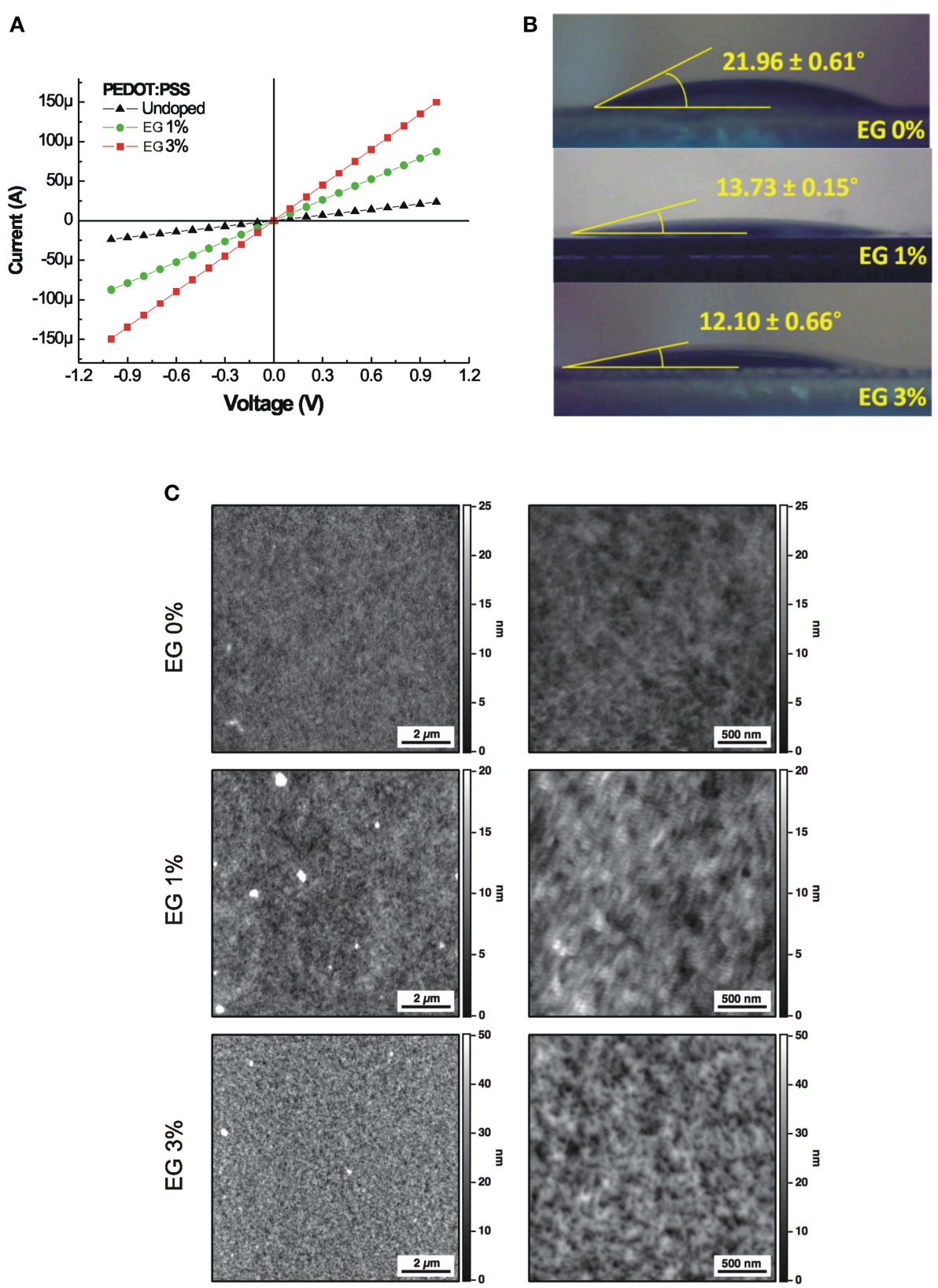

FIGURE 1 | (A) Current-Voltage characteristics of the PEDOT:PSS layers doped with different concentration of EG. (B) Water contact angles of the PEDOT:PSS layers doped with different concentration of EG. (C) $10 \times 10 \mu \mathrm{m}^{2}$ AFM images (left column) and the corresponding $2.5 \times 2.5 \mu \mathrm{m}^{2} \mathrm{magnifications}$ (right column) of PEDOT:PSS layers doped with increasing amounts of EG $(0,1$, and $3 \%)$. Brighter color areas in the image correspond to higher portions of the sample.

neuronal health (Carp, 1992; Djuric et al., 2015; Gao et al., 2015).

Neurons grown on PEDOT:PSS layers did not show any statistically significant differences with respect to the control in these values (resting membrane potential: $-45 \pm 4,-47 \pm 3$, and $-46 \pm 3 \mathrm{mV}$; capacitance: $71 \pm 19,63 \pm 26$, and $59 \pm 6 \mathrm{pF}$; input resistance: $740 \pm 98,857 \pm 98,855 \pm 119 \mathrm{M} \Omega$, respectively control $n=16$, undoped PEDOT:PSS $n=18$, PEDOT:PSS $1 \%$ $\mathrm{EG} n=15$; Figure 2C).
Neurons grown on PEDOT:PSS layers showed spontaneous synaptic activity made up by a mixed population of postsynaptic currents (detected as inward currents in our recording conditions; Figure 2D; Cellot et al., 2011) indicating that such substrates support the growth of neurons connected by functionally active synapses. sPSCs amplitude and frequency on PEDOT:PSS did not differ from control ones (sPSCs amplitude: $50 \pm 5,49 \pm 8$, and $50 \pm 7 \mathrm{pA}$; sPSCs frequency: $1.4 \pm 0.2,1.8 \pm 0.5$, and $1.9 \pm 0.4 \mathrm{~Hz}$ for control $n=17$ 
A

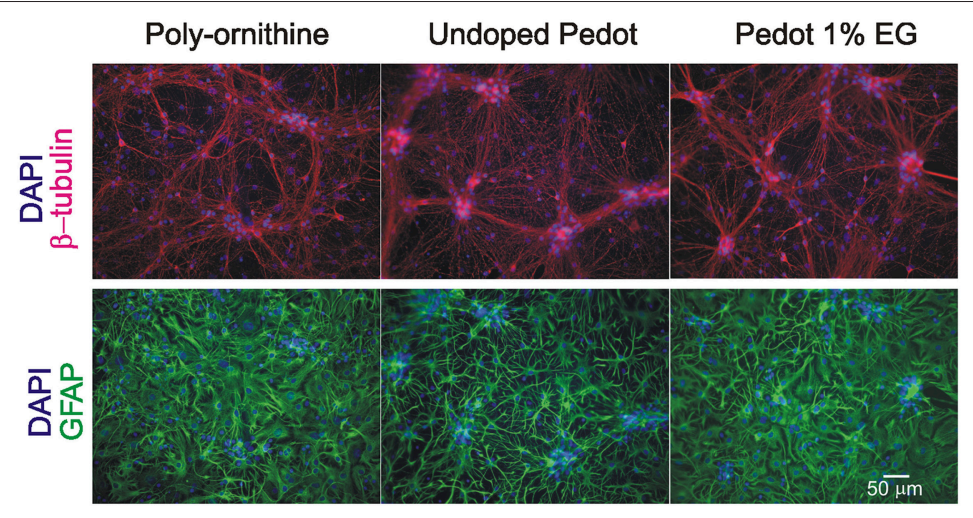

B
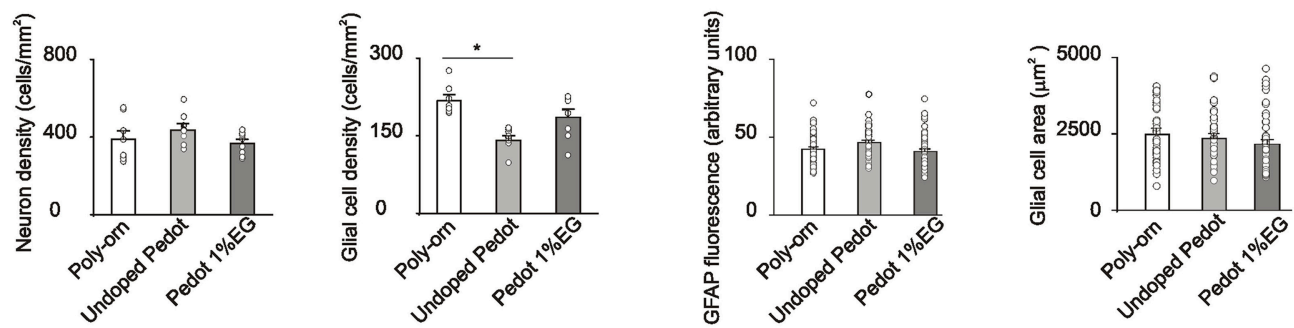

C

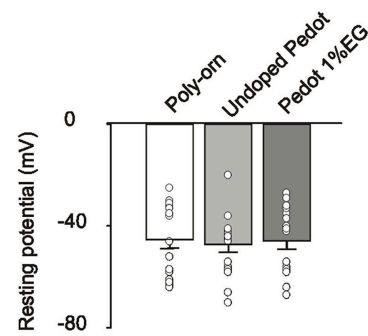

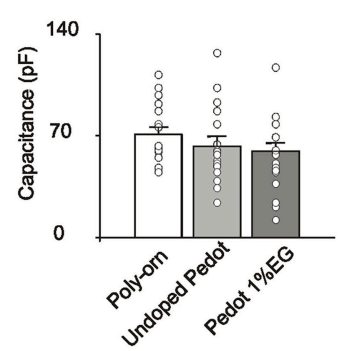

D Poly-ornithine

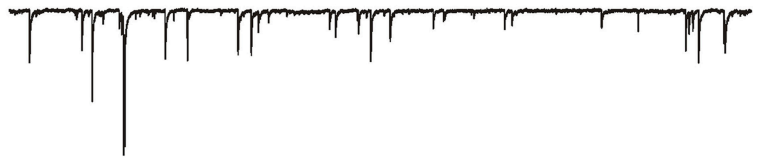

Undoped Pedot

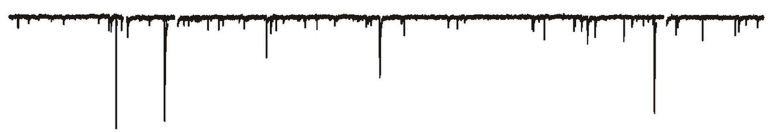

Pedot 1\% EG

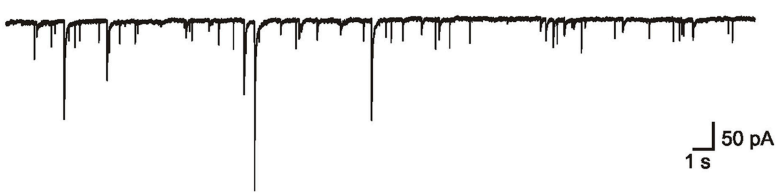

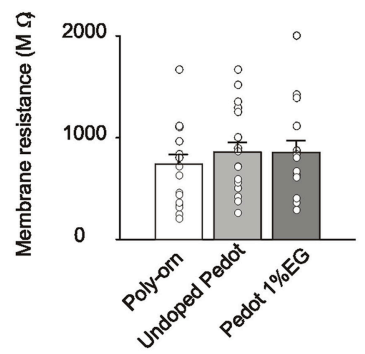
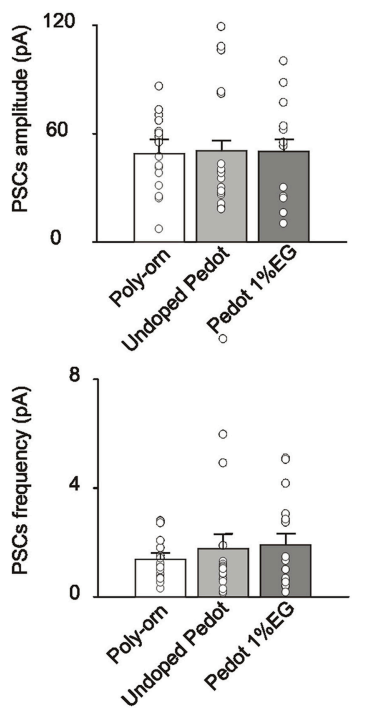

FIGURE 2 | (A) Immunofluorescence images of cultures grown on poly-ornithine, undoped PEDOT:PSS (O\% EG) and PEDOT:PSS 1\% EG. Cells were stained with antibodies anti- $\beta$-tubulin (red), GFAP (green) and nuclei were marked with DAPI (blue) (B). Bar plots show mean values of neuronal and glial cells density, GFAP fluorescence intensity and glial cells areas in the different conditions of growth. Superimposed dots to the bars are values from single fields (for cell density) or from single cells (for GFAP intensity and glial cell areas). Note that the glial cell density is significantly decreased on undoped PEDOT:PSS respect to the poly-ornithine control $\left({ }^{*} p<0.05, T\right.$-test). (C) Bar plots are mean values of resting membrane potentials, capacitance and membrane resistance. Superimposed dots to the bars are values from single experiments. (D) On the left, exemplificative recordings from neurons grown on poly-ornithine, undoped PEDOT:PSS and PEDOT:PSS 1\% EG respectively. On the right, bar plots show the means values of spontaneous PSCs amplitude and frequency in the different conditions of growth. Superimposed dots to the bar are values from single experiments. 
undoped PEDOT:PSS $n=18$ and PEDOT:PSS 1\% EG $n=16$; Figure 2D). These results further support the similar size of control and PEDOT:PSS neuronal networks after 1 week of in vitro growth.

\section{Long Term Hippocampal Cultures on 1\% EG and 3\% EG PEDOT:PSS}

PEDOT:PSS containing EG has higher electrical conductivity than EG free one (Wang et al., 2005; Nardes et al., 2008), thus, in view of the potential of this material for prosthetic applications, we tested neurons seeded on PEDOT:PSS 1 and 3\% $\mathrm{EG}$, to verify the viability of long term cultures on PEDOT:PSS layers.

After $21 \mathrm{DIV}$ (Figure 3A) neuronal density did not differ between the PEDOT:PSS and controls $(249 \pm 33,256 \pm 34$, and $234 \pm 20$ cells $/ \mathrm{mm}^{2}$, respectively for control, PEDOT:PSS $1 \%$ EG and PEDOT:PSS 3\% EG, $n=8$ fields each). Conversely, in PEDOT:PSS GFAP-positive glial cells were significantly reduced upon 3 weeks of culturing ( $65 \pm 4$ cells $/ \mathrm{mm}^{2}$ in control, $42 \pm 4$ cells $/ \mathrm{mm}^{2}$ on PEDOT:PSS $1 \%$ EG and $45 \pm 6$ cells $/ \mathrm{mm}^{2}$ on PEDOT:PSS 3\% EG; $p<0.05, T$-Test, Figure 3B).

In these samples GFAP fluorescence intensity was found to be similar among the various conditions of growth (GFAP fluorescence: control $69 \pm 3.2$ arbitrary units, PEDOT:PSS 1\% EG $73 \pm 3.7$ arbitrary units and PEDOT:PSS 3\% EG $78 \pm 4$ arbitrary units); however GFAP-positive cell area values in PEDOT:PSS in PEDOT:PSS were significantly smaller with respect to controls (glial cells area: control 11,882 $\pm 758 \mu \mathrm{m}^{2}$, PEDOT:PSS $1 \%$ EG $9018 \pm 1209 \mu \mathrm{m}^{2}$ and PEDOT:PSS 3\% EG $7053 \pm 633 \mu \mathrm{m}^{2}$, $n=56, n=37, n=37$, respectively; $p<0.05$, T-Test, Figure 3B).

Similar to what detected in 8 DIV cultures, the membrane passive properties of sampled neurons did not differ among the three groups showing on average a resting membrane potential of $-48 \pm 2,-46 \pm 2$, and $-45 \pm 2 \mathrm{mV}$; capacitance values of $70 \pm$ $8,68 \pm 6$, and $59 \pm 6 \mathrm{pF}$ and input resistance values of $414 \pm 55$, $302 \pm 34,464 \pm 90 \mathrm{M} \Omega$, in control $n=25$, PEDOT:PSS $1 \%$ EG $n=25$, PEDOT:PSS $3 \%$ EG $n=24$, respectively (Figure 3C).

In the very same conditions, $21 \mathrm{DIV}$, the measured sPSC frequency and amplitude were unaltered in PEDOT:PSS layers in respect to age matched controls (sPSCs amplitude: $71 \pm 9,81 \pm$ 12 , and $69 \pm 10 \mathrm{pA}$; sPSCs frequency: $5.6 \pm 1.3,5.3 \pm 1.4$, and $4.6 \pm 1.3 \mathrm{~Hz}$ for control $n=25$, PEDOT:PSS $1 \%$ EG $n=25$ and PEDOT:PSS $3 \%$ EG $n=24$; Figure 3D).

\section{SEM Images Show Healthy Morphology of Neurons Grown on PEDOT:PSS Layers}

Figure 4A shows neuronal network visualized by low magnification SEM microscopy. Darker areas correspond to glial cells. Higher magnification of micrographs (Figure 4B) shows the healthy appearance of single neurons when developed on PEDOT:PSS layers, similar to that of controls. In all the conditions of growth, 3-4 neurites (see methods) emerged from the soma and extend centrifugally on the substrates, organized in intricate arborizations.

\section{DISCUSSION}

We studied PEDOT:PSS growth substrates in long-term hippocampal cultures, in the perspective of exploiting such materials for neural prosthesis. Implantable electrodes technology is continuously evolving also in terms of the chemistry of the material used, to improve the device longevity and performance. In particular, highly conductive materials should be design also to minimize tissue damage and reduce glial reactions (Lempka et al., 2009). In this framework, we tested primary hippocampal cells cultured interfaced to PEDOT:PSS doped with different concentrations of EG $(0,1$, and $3 \%)$, a dopant reported to increase the electrical properties of conductive polymers (Crispin et al., 2003; Ouyang et al., 2004, 2015; Romeo et al., 2015). We describe here for the first time that EG not only affects the conductivity of the layers, but also improves wettability and roughness, and selectively reduces glial cell reaction. Importantly, neuronal maturation, synapse formation and function are unaltered by these substrates.

We confirmed the linear relationship between the conductive properties of PEDOT:PSS and the amount of EG dopant. In addition, the analysis of the static contact angle on the PEDOT:PSS layers indicated the ability of EG in controlling the hydrophilicity of the surface. The electrical conductivity and the wettability of the PEDOT:PSS layers are two fundamental properties for developing long-term implants, in fact high electrical conductivity allows an improved transport of the neural electrical signals and the higher wettability makes the PEDOT:PSS surfaces more hydrophilic and therefore suitable for the adhesion of living cells.

The roughness analysis of PEDOT:PSS layers showed an increase in surface RMS values as the amount of EG was increased. Compared to the control 0\% EG, PEDOT:PSS 1\% EG shows an increase in roughness of about $37 \%$ while PEDOT:PSS $3 \%$ EG presents a nearly three times higher roughness (287\%). $\mathrm{S}_{\mathrm{r}}$ ratio between the effective surface area and the projected area presents a small decrease in PEDOT:PSS 1\% EG samples than 0\% EG controls, but PEDOT:PSS 3\% shows a valuable increase of the exposed area (about 3\% larger than controls, see Results). This may potentially lead to improved charge exchange efficacy in the PEDOT:PSS 3\% EG.

The biocompatibility of PEDOT:PSS was tested by culturing dissociated postnatal hippocampal cells isolated from neonatal rats. This is a widely adopted and characterized in vitro model for neuronal networks formation and for synaptic physiology studies. Neurons, after a few hours from seeding, are able to attach to the substrates, if permissive, and begin to extend neurites. In the following days, neurites elongate, take contact with other neurons, forming synapses that are functionally active after a week in vitro. Spontaneous synaptic activity comprises both excitatory and inhibitory postsynaptic currents as observed in vivo (Segal, 1983; Köller et al., 1990; Siebler et al., 1993). By SEM, we observed that neurons grown on PEDOT:PSS layers show a healthy morphology, with many neurites extending from the soma and forming arborizations, similarly to cells on control substrates. Also the size of the network was comparable, as immunofluorescence experiments show, where the density of 


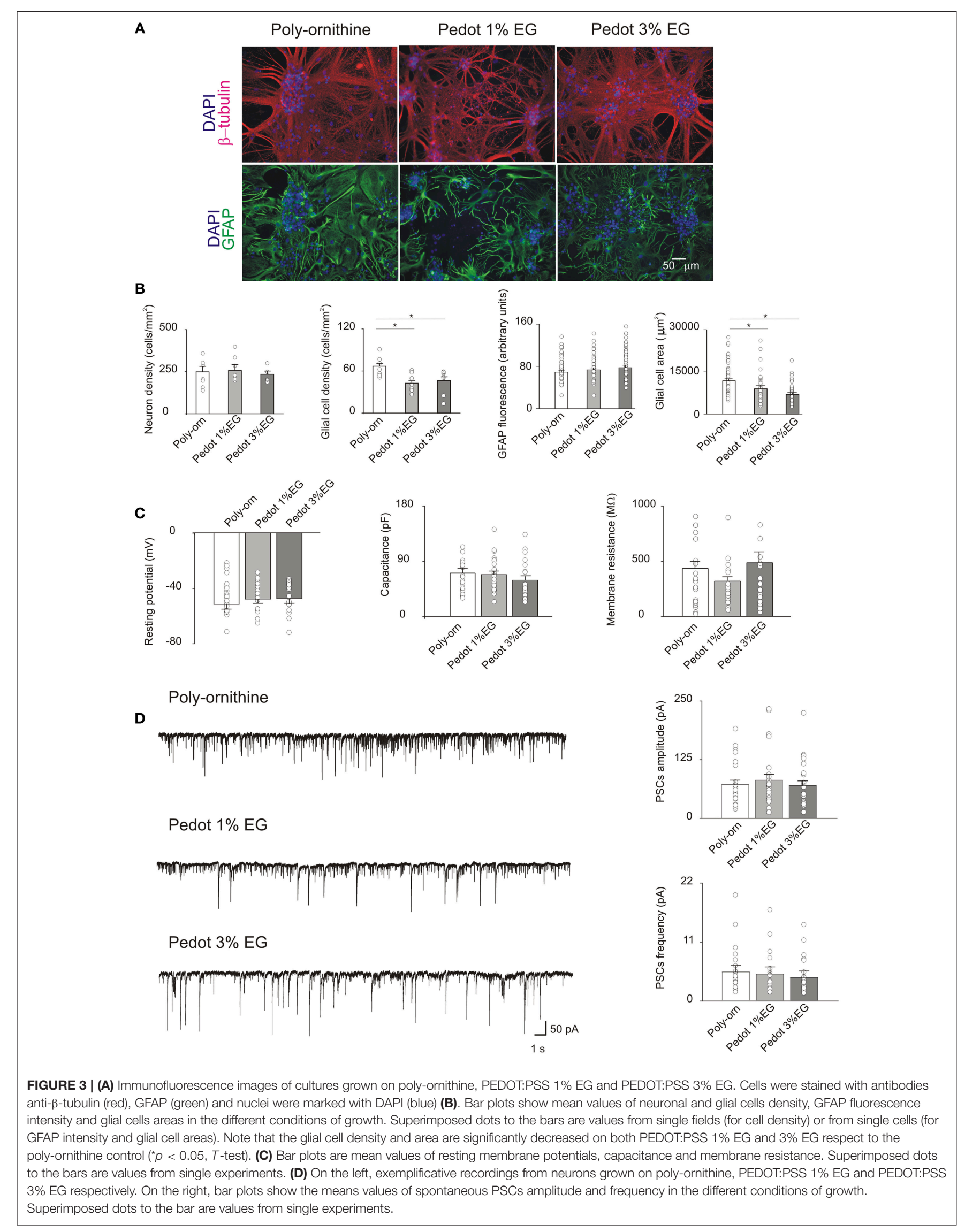



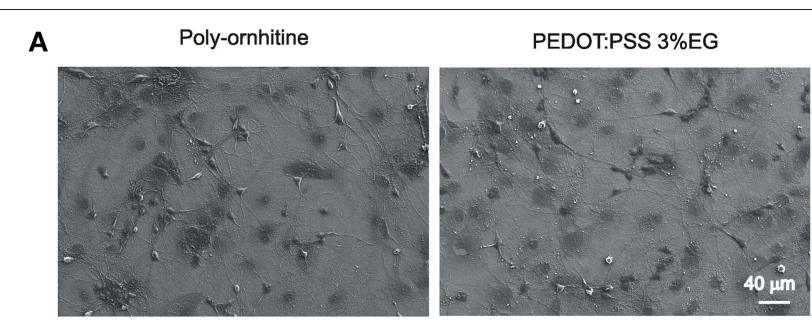

B

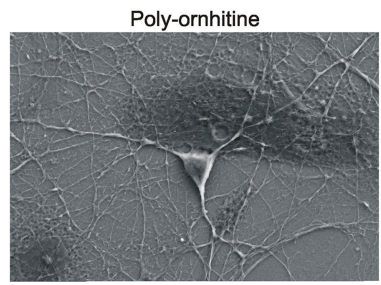

PEDOT:PSS $1 \%$ EG
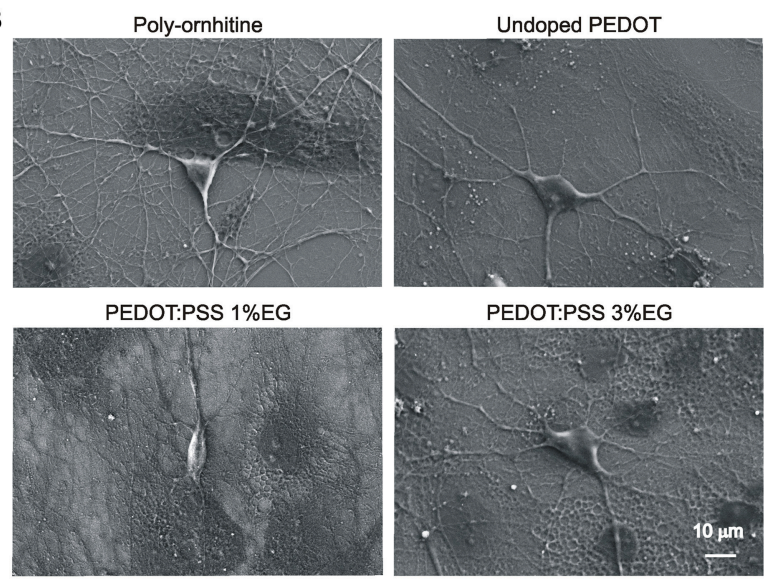

PEDOT:PSS 3\%EG

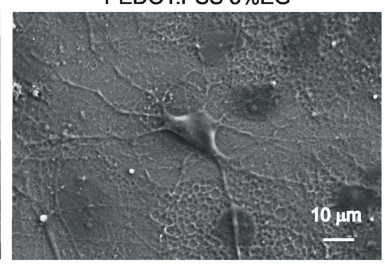

FIGURE 4 | Scanning electron microscopy micrographs showing hippocampal cultures grown on PEDOT:PSS layers. (A) Lower magnification micrographs display that qualitatively the size of neuronal network is comparable between undoped PEDOT:PSS and PEDOT:PSS 3\% EG. (B) Higher magnification micrographs show the healthy morphology of single neurons grown on the different substrates.

neurons that attached and developed on PEDOT:PSS substrates was similar to that of control, both at 1 and 3 weeks in vitro.

We further characterized neurons developed on PEDOT:PSS from a functional point of view via electrophysiological recordings (Lovat et al., 2005; Cellot et al., 2011). Although electrical activity from neuronal populations grown on conductive polymers has been partially characterized previously by MEAs (Nyberg et al., 2007), in our study, by single cell patch clamp technique, we measured the single neuron membrane properties as well as those of single synaptic events.

Our experiments revealed that single cell membrane passive properties, known indicators of neuronal health (Carp, 1992; Djuric et al., 2015; Gao et al., 2015), and the spontaneous activity of the network, measured in terms of amplitude and frequency of sPSCs, were fully comparable between cultures grown on PEDOT:PSS and controls. This was observed for neurons after a week in culture (undoped and 1\% EG), but also in longterm cultures ( 3 weeks in vitro) with even higher concentrations of dopant (up to 3\% EG). Thus, on PEDOT:PSS interfaces we develop healthy neurons and functionally active synaptic networks, comparable to those grown on traditional peptidelayered substrates (controls).
Intriguingly, PEDOT:PSS down-regulated glial cells, with a partial reduction detected also after 1 week of culturing. The GFAP-positive glia shows clear reduction in cell density and glial cells have smaller GFAP-positive area after being interfaced for 3 weeks on PEDOT:PSS, with respect to control. Notably, the intensity of GFAP fluorescence signal appeared similar among all tested groups.

An increased number of glial cells and an enhanced glial cellular size are usually associated with pathological states (Yang and Wang, 2015), thus PEDOT:PSS apparently reduces glial response, a relevant feature for exploiting electrode-material able to induce low glial reactions (Lempka et al., 2009).

Other conductive polymers, such as PEDOP and P3MT, but not PEDOT, were reported to reduce astrocyte responses (Forcelli et al., 2012). However, in the PEDOT layers reported by Forcelli et al. (2012) PSS was not used as counter ion dopant and the deposition process was also different, probably accounting for the different results obtained.

It is interesting to note that the reduced glial response observed on PEDOT:PSS layers in our long-term cultures seems not to influence the functional properties of neuronal networks, as our experiments detected similar neuronal density and comparable electrical activity between PEDOT:PSS and controls.

It is possible that the neuronal network developed on PEDOT substrates in the presence of a reduced number of glial cells could keep intact its functional properties thanks to the hydrophilic nature of the material. This would allow the accumulation of extracellular matrix proteins (Van Kooten et al., 1992), creating a favorable environment for neuronal growth (Masuda-Nakagawa and Wiedemann, 1992).

Thus, our findings suggest that PEDOT:PSS materials can be exploited for the development of new implantable devices. We speculate that such materials in vivo can control the glial reactivity, with limited effects on neuronal viability. Certainly this hypothesis requires being validated trough further experiments.

\section{AUTHOR CONTRIBUTIONS}

GC performed cell biology, electrophysiology and immunofluorescence experiments and analysis; PL, GT, FF, SI, MP, and GS contributed to material design, production and characterization; DS performed SEM and AFM experiments; LB and GS conceived the study; LB conceived the biological experimental design; GS, MP, and LB provided funding; GC and $\mathrm{LB}$ wrote the manuscript. All authors have given approval to the final version of the manuscript.

\section{FUNDING}

We acknowledge financial support from the NEURO SCAFFOLDS-FP7-NMP-604263 and PRIN-MIUR n. 2012 MYESZW. 


\section{REFERENCES}

Baek, S., Green, R. A., and Poole-Warren, L. A. (2014). Effects of dopants on the biomechanical properties of conducting polymer films on platinum electrodes. J. Biomed. Mater. Res. A 102, 2743-2754. doi: 10.1002/jbm.a.34945

Berggren, M., and Richter-Dahlfors, A. (2007). Organic bioelectronics. Adv. Mater. 19, 3201-3213. doi: 10.1002/adma.200700419

Carp, J. S. (1992). Physiological properties of primate lumbar motoneurons. J. Neurophysiol. 68, 1121-1132.

Cellot, G., Toma, F. M., Varley, Z. K., Laishram, J., Villari, A., Quintana, M., et al. (2011). Carbon nanotube scaffolds tune synaptic strength in cultured neural circuits: novel frontiers in nanomaterial-tissue interactions. J. Neurosci. 31, 12945-12953. doi: 10.1523/JNEUROSCI.133211.2011

Chen, Y., He, B., Lee, J., and Patankar, N. A. (2005). Anisotropy in the wetting of rough surfaces. J. Colloid Interface Sci. 281, 458-464. doi: 10.1016/j.jcis.2004.07.038

Collazos-Castro, J. E., Polo, J. L., Hernández-Labrado, G. R., Padial-Cañete, V., and García-Rama, C. (2010). Bioelectrochemical control of neural cell development on conducting polymers. Biomaterials 31, 9244-9255. doi: 10.1016/j.biomaterials.2010.08.057

Crispin, X., Marciniak, S., Osikowicz, W., Zotti, G., van der Gon, A. W. D., Louwet, F., et al. (2003). Conductivity, morphology, interfacial chemistry, and stability of poly(3,4-ethylene dioxythiophene)-poly(styrene sulfonate): a photoelectron spectroscopy study. J. Polym. Sci. B Polym. Phys. 41, 2561-2583. doi: $10.1002 /$ polb.10659

Cui, X., Wiler, J., Dzaman, M., Altschuler, R. A., and Martin, D. C. (2003). In vivo studies of polypyrrole/peptide coated neural probes. Biomaterials 24, 777-787. doi: 10.1016/S0142-9612(02)00415-5

Dimitrieva, O. P., Grinkoa, D. A., Noskovb Yu, V., Ogurtsovb, N. A., and Pudb, A. A. (2009). PEDOT:PSS films effect of organic solvent additives and annealing on the film conductivity. Synth. Met. 159, 2237-2239. doi: 10.1016/j.synthmet.2009.08.022

Djuric, U., Cheung, A. Y., Zhang, W., Mok, R. S., Lai, W., Piekna, A., et al. (2015). MECP2e1 isoform mutation affects the form and function of neurons derived from Rett syndrome patient iPS cells. Neurobiol. Dis. 76, 37-45. doi: 10.1016/j.nbd.2015.01.001

Donoghue, J. P. (2002). Connecting cortex to machines: recent advances in brain interfaces. Nat. Neurosci. 5, 1085-1088. doi: 10.1038/nn947

Fabbro, A., Villari, A., Laishram, J., Scaini, D., Toma, F. M., Turco, A., et al. (2012). Spinal cord explants use carbon nanotube interfaces to enhance neurite outgrowth and to fortify synaptic inputs. ACS Nano 6, 2041-2055. doi: $10.1021 / \mathrm{nn} 203519 \mathrm{r}$

Forcelli, P. A., Sweeney, C. T., Kammerich, A. D., Lee, B. C., Rubinson, L. H., Kayinamura, Y. P., et al. (2012). Histocompatibility and in vivo signal throughput for PEDOT, PEDOP, P3MT, and polycarbazole electrodes. J. Biomed. Mater. Res. A 100, 3455-3462. doi: 10.1002/jbm.a. 34285

Gao, Y., Liu, L., Li, Q., and Wang, Y. (2015). Differential alterations in the morphology and electrophysiology of layer II pyramidal cells in the primary visual cortex of a mouse model prenatally exposed to LPS. Neurosci. Lett. 591, 138-143. doi: 10.1016/j.neulet.2015.02.043

Goodrick, S. (2014). Man and machine. Lancet Neurol. 13:1080. doi: 10.1016/S1474-4422(14)70247-9

Green, R. A., Lovell, N. H., Wallace, G. G., and Poole-Warren, L. A. (2008). Conducting polymers for neural interfaces: challenges in developing an effective long-term implant. Biomaterials 29, 3393-3399. doi: 10.1016/j.biomaterials.2008.04.047

Gunasekera, B., Saxena, T., Bellamkonda, R., and Karumbaiah, L. (2015). Intracortical recording interfaces: current challenges to chronic recording function. ACS Chem. Neurosci. 6, 68-83. doi: 10.1021/cn50 02864

Heeger, A. J. (2001). Semiconducting and metallic polymers: the fourth generation of polymeric materials. Angew. Chem. Int. Ed Engl. 40, 2591-2611. doi: 10.1002/1521-3773(20010716)40:14<2591::AID-ANIE2591>3.0.CO;2-0

Henke, L., Nagy, N., and Krull, U. J. (2002). An AFM determination of the effects on surface roughness caused by cleaning of fused silica and glass substrates in the process of optical biosensor preparation. Biosens. Bioelectron. 17, 547-555. doi: 10.1016/S0956-5663(02)00012-X

Khodagholy, D., Doublet, T., Gurfinkel, M., Quilichini, P., Ismailova, E., Leleux, P., et al. (2011). Highly conformable conducting polymer electrodes for in vivo recordings. Adv. Mater. 23, 268-272. doi: 10.1002/adma.201102378

Khodagholy, D., Gelinas, J. N., Thesen, T., Doyle, W., Devinsky, O., Malliaras, G. G., et al. (2015). NeuroGrid: recording action potentials from the surface of the brain. Nat. Neurosci. 18, 310-315. doi: 10.1038/nn.3905

Khodagholy, D., Rivnay, J., Sessolo, M., Gurfinkel, M., Leleux, P., Jimison, L. H., et al. (2013). High transconductance organic electrochemical transistors. Nat. Commun. 4, 2133. doi: 10.1038/ncomms3133

Kim, Y. H., Sachse, C., Machala, M. L., May, C., Müller-Meskamp, L., and Leo, K. (2011). Highly conductive PEDOT:PSS electrode with optimized solvent and thermal post-treatment for ITO-free organic solar cells. Adv. Funct. Mat. 21, 1076-1081. doi: 10.1002/adfm.201002290

Köller, H., Siebler, M., Schmalenbach, C., and Müller, H. W. (1990). GABA and glutamate receptor development of cultured neurons from rat hippocampus, septal region, and neocortex. Synapse 5, 59-64. doi: 10.1002/syn.890050105

Lempka, S. F., Miocinovic, S., Johnson, M. D., Vitek, J. L., and McIntyre, C. C. (2009). In vivo impedance spectroscopy of deep brain stimulation electrodes. J. Neural Eng. 6:046001. doi: 10.1088/1741-2560/6/4/046001

Lovat, V., Pantarotto, D., Lagostena, L., Cacciari, B., Grandolfo, M., Righi, M., et al. (2005). Carbon nanotube substrates boost neuronal electrical signaling. Nano Lett. 5, 1107-1110. doi: 10.1021/nl050637m

Ludwig, K. A., Uram, J. D., Yang, J., Martin, D. C., and Kipke, D. R. (2006). Chronic neural recordings using silicon microelectrode arrays electrochemically deposited with a poly(3,4-ethylenedioxythiophene) (PEDOT) film. J. Neural Eng. 3, 59-70. doi: 10.1088/1741-2560/3/1/007

Machado, S., Araújo, F., Paes, F., Velasques, B., Cunha, M., Budde, H., et al. (2010). EEG-based brain-computer interfaces: an overview of basic concepts and clinical applications in neurorehabilitation. Rev. Neurosci. 21, 451-468. doi: 10.1515/revneuro.2010.21.6.451

Masuda-Nakagawa, L. M., and Wiedemann, C. (1992). The role of matrix molecules in regeneration of leech CNS. J. Neurobiol. 23, 551-567. doi: $10.1002 /$ neu. 480230509

Mazzatenta, A., Giugliano, M., Campidelli, S., Gambazzi, L., Businaro, L., Markram, H., et al. (2007). Interfacing neurons with carbon nanotubes: electrical signal transfer and synaptic stimulation in cultured brain circuits. J. Neurosci. 27, 6931-6936. doi: 10.1523/JNEUROSCI.1051-07.2007

Nardes, A. M., Janssen, R. A. J., and Kemerink, M. A. (2008). Morphological model for the solvent-enhanced conductivity of PEDOT:PSS thin films. Adv. Funct. Mater. 18, 865-871. doi: 10.1002/adfm.200700796

Nečas, D., and Klapetek, P. (2013). One-dimensional autocorrelation and power spectrum density functions of irregular regions. Ultramicroscopy 124, 13-19. doi: 10.1016/j.ultramic.2012.08.002

Nyberg, T., Shimada, A., and Torimitsu, K. (2007). Ion conducting polymer microelectrodes for interfacing with neural networks. J. Neurosci. Methods 160, 16-25. doi: 10.1016/j.jneumeth.2006.08.008

Ouyang, J., X, Chu, C.-W., Yang, Y., Li,G., and Shinar, J. (2004). On the mechanism of conductivity enhancement in poly(3,4 ethylenedioxythiophene): poly(styrene-sulfonate) film through solvent treatment. Polymer 45 8443-8450. doi: 10.1016/j.polymer.2004.10.001

Ouyang, L., Musumeci, C., Jafari, M. J., Ederth, T., and Inganäs, O. (2015). Imaging the phase separation between PEDOT and polyelectrolytes during processing of highly conductive PEDOT:PSS films. ACS Appl. Mater. Interfaces 7, 19764-19773. doi: 10.1021/acsami.5b05439

Palchesko, R. N., Zhang, L., Sun, Y., and Feinberg, A. W. (2012). Development of polydimethylsiloxane substrates with tunable elastic modulus to study cell mechanobiology in muscle and nerve. PLOS ONE 7:e51499. doi: 10.1371/journal.pone.0051499

Polikov, V. S., Tresco, P. A., and Reichert, W. M. (2005). Response of brain tissue to chronically implanted neural electrodes. J. Neurosci. Methods 148, 1-18. doi: 10.1016/j.jneumeth.2005.08.015

Richardson-Burns, S. M., Hendricks, J. L., Foster, B., Povlich, L. K., Kim, D. H., and Martin, D. C. (2007). Polymerization of the conducting polymer poly(3,4ethylenedioxythiophene) (PEDOT) around living neural cells. Biomaterials 28, 1539-1552. doi: 10.1016/j.biomaterials.2006.11.026 
Romeo, A., Tarabella, G., D’Angelo, P., Caffarra, C., Cretella, D., Alfieri, R., et al. (2015). Drug-induced cellular death dynamics monitored by a highly sensitive organic electrochemical system. Biosens. Bioelectron. 68, 791-797. doi: 10.1016/j.bios.2015.01.073

Salazar, M., Pariente, J. A., Salido, G. M., and González, A. (2008). Ebselen increases cytosolic free $\mathrm{Ca}^{2+}$ concentration, stimulates glutamate release and increases GFAP content inrat hippocampal astrocytes. Toxicology 244, 280-291. doi: 10.1016/j.tox.2007.12.002

Schmidt, C. E., Shastri, V. R., Vacanti, J. P., and Langer, R. (1997). Stimulation of neurite outgrowth using an electrically conducting polymer. Proc. Natl. Acad. Sci. U.S.A. 94, 8948-8953. doi: 10.1073/pnas.94.17.8948

Segal, M. J. (1983). Rat hippocampal neurons in culture: responses to electrical and chemical stimuli. Neurophysiol 50, 1249-1264.

Siebler, M., Köller, H., Stichel, C. C., Müller, H. W., and Freund, H. (1993). Spontaneous activity and recurrent inhibition in cultured hippocampal networks. Synapse 14, 206-213. doi: 10.1002/syn.890140304

Tamburri, E., Orlanducci, S., Toschi, F., Terranova, M. L., and Passeri, D. (2009). Growth mechanisms, morphology, and electroactivity of PEDOT layers produced by electrochemical routes in aqueous medium. Synth. Met. 159, 406-414. doi: 10.1016/j.synthmet.2008.10.014

Tarabella, G., Pezzella, A., Romeo, A., D’Angelo, P., Coppedè, N., Calicchio, M., et al. (2013). Irreversible evolution of eumelanin redox states detected by an organic electrochemical transistor: en route to bioelectronics and biosensing. J. Mater. Chem. 1, 3843-3849. doi: 10.1039/c3tb 20639d

Van Kooten, T. G., Schakenraad, J. M., van der Mei, H. C., van der Mei, H. J. (1992). Influence of substratum wettability on the strength of adhesion of human fibroblasts. Biomaterials 13, 897-904. doi: 10.1016/0142-9612(92) 90112-2
Wang, T., Qi, Y., Xu, J., Hu, X., and Chen, P. (2005). Effects of poly(ethylene glycol) on electrical conductivity of poly(3,4-ethylenedioxythiophene)poly(styrenesulfonic acid) film. Appl. Surf. Sci. 250, 188-194. doi: 10.1016/j.apsusc.2004.12.051

Williamson, A., Rivnay, J., Kergoat, L., Jonsson, A., Inal, S., Uguz, I., et al. (2015). Controlling epileptiform activity with organic electronic ion pumps. Adv. Mater. 27, 3138-3144. doi: 10.1002/adma.201500482

Xiao, Y., Martin, D. C., Cui, X., and Shenai, M. (2006). Surface modification of neural probes with conducting polymer poly(hydroxymethylated-3,4ethylenedioxythiophene) and its biocompatibility. Appl. Biochem. Biotechnol. 128, 117-130. doi: 10.1385/ABAB:128:2:117

Yamato, H., Ohwa, M., and Wernet, W. (1995). Stability of polypyrrole and poly(3,4-ethylene dioxythiophene) for biosensor application. J. Electroanal. Chem. 397, 163-170. doi: 10.1016/0022-0728(95)04156-8

Yang, Z., and Wang, K. K. (2015). Glial fibrillary acidic protein: from intermediate filament assembly and gliosis to neurobiomarker. Trends Neurosci. 38, 364-374. doi: 10.1016/j.tins.2015.04.003

Conflict of Interest Statement: The authors declare that the research was conducted in the absence of any commercial or financial relationships that could be construed as a potential conflict of interest.

Copyright (c) 2016 Cellot, Lagonegro, Tarabella, Scaini, Fabbri, Iannotta, Prato, Salviati and Ballerini. This is an open-access article distributed under the terms of the Creative Commons Attribution License (CC BY). The use, distribution or reproduction in other forums is permitted, provided the original author(s) or licensor are credited and that the original publication in this journal is cited, in accordance with accepted academic practice. No use, distribution or reproduction is permitted which does not comply with these terms. 